\title{
Eosinophilic Ureteritis Causing Ureterohydronephrosis: A Case Report
}

\section{Uspal Bahadur Bajracharya ${ }^{1}$, Anil Kumar Sah', Hari Baral', Ghanasham Sigdel ${ }^{1}$, Guna Kumar Shrestha ${ }^{2}$ and Rojan Adhikari ${ }^{1}$}

${ }^{1}$ Department of Urology, Manmohan Memorial Medical College and Teaching Hospital, Kathmandu, Nepal ${ }^{2}$ Department of Urology, Nepal Mediciti Hospital, Lalitpur, Nepal

\footnotetext{
ABSTRACT

Introduction: Eosinophilic ureteritis is a rare cause of ureteric stricture causing hydroureteronephrosis. The exact aetiology of this condition is still unknown. This condition has been described in relation to atopy, hypereosinophilic syndrome and prior ureteral interventions. The histopathology is the conclusive diagnostic modality. The surgical resection of the ureteric stricture part with end-to-end anastomosis is usually successful. Herein, we report a case of a 19-year-old woman, who presented with right flank pain. With imaging and diagnostic ureteroscopy, the case was diagnosed as eosinophilic ureteritis. The case was successfully managed with resection of the stenosing part and end-to-end anastomosis. No cases of the disease have been reported until now in Nepal.

Key words: Eosinophilic Ureteritis; Flank Pain; Hydronephrosis; Ureteroscopy
}

Correspondence: Anil Kumar Sah, FCPS Resident Urology, Department of Urology, Nepal Mediciti Hospital, Kathmandu, Nepal. E-mail: anil.frens@gmail.com

DOI: $10.3126 / \mathrm{mjsbh} . v 20 \mathrm{i} 2.36632$

Submitted on: $2021-04-26$

Accepted on: 2021-06-07

This work is licensed under creative common license:

http://creativecommons.org/licenses/by-nc-nd/4.0/ @ MJSBH 2020 


\section{INTRODUCTION}

Flank pain is a common presentation of urological emergency. An acute ureteral obstruction from impacted stone is perhaps the most common cause. ${ }^{1}$ Flank pain due to eosinophilic ureteritis is a rare finding. ${ }^{2}$ We report a case of a 19 -year-old woman presenting with flank pain with features of urinary stone disease. It could be due to a variety of factors, with a high chance to be overlooked. As clinical and imaging studies are not specific, high index of suspicion is necessary to the proper diagnosis and appropriate management. ${ }^{3}$ No cases of the disease have been reported until now in Nepal.

\section{CASE REPORT}

A 19-year-old married woman presented with the complaint of dull aching in the right flank for past 15 days. The pain was continuous, non-radiating and increasing. However, there was no history of fever, hematuria or dysuria. There was also no other significant past medical history. She denied the history of past renal stone disease, endourological intervention or abdominal or pelvic trauma. She had a normal menstrual history. The physical examination revealed the right renal angle tenderness and no other significant abnormality. Urine analysis revealed packed pus cells per High Power Field (HPF), 3 - 4 red blood cells / HPF, and $3+$ protein. Her baseline investigations were within the normal limits with normal serum creatinine and normal eosinophil count in blood. The urine culture showed no growth of pathogen with Acid Fast Stain showing no bacilli.

The ultrasonography (USG) of abdomen and pelvis revealed thickening of right proximal ureteric wall of around one to two $\mathrm{cm}$ with mild right hydroureteronephrosis (Figure 1). The CT-IVU revealed mild hydronephrosis of right kidney with dilated proximal ureter with abrupt tapering of proximal ureter suggestive of right ureteric stricture (Figure 2a, 2b). A pre-operative working diagnosis of right proximal ureteric stricture and right mild hydronephrosis was made.

The patient underwent Right Diagnostic Ureterorenoscopy (URS) under general anaesthesia with $6.5 / 8.5 \mathrm{Fr}$ semi rigid ureteroscope (wolf). The scope was negotiated up to right mid ureter but further negotiation at the level of proximal ureter was not possible. Intraoperative Retrograde Pyelogram (RPG) showed proximal ureteric stricture. Right sided Double-J (DJ) ureteral stent negotiation was also unsuccessful. After informed consent taken from patient party, the case was converted to open surgical exploration in the same sitting. It revealed dilated proximal ureter with 2.5 $\mathrm{cm}$ of stricture at upper ureter approximately 7.5 $\mathrm{cm}$ from pelvic-ureteric junction. Excision of the stricture segment and end-to-end ureteroureterostomy was performed over internal DJ stenting in a tension-free manner. Microscopy of the excised specimen revealed dominant eosinophilic infiltration along with lymphocytes and neutrophils (Figure 3a). There was subepithelial hyalinisation edema. Congested blood vessels were seen in adventitia (Figure 3b). A diagnosis of eosinophilic ureteritis was made based on this pathological finding. She had uneventful recovery. Double J stent was removed after six weeks. We followed her with upper tract imaging, which was normal.

\section{DISCUSSION}

Eosinophilic ureteritis is a rare stenosing condition of the ureter, which can cause flank pain and / or unilateral hydronephrosis. ${ }^{1}$ Singh et al reported a case of bilateral eosinophilic ureteritis, possibly triggered by filariasis. ${ }^{2}$ Spark et al reported the relation of this condition with atopy, hypereosinophilic syndromes and trauma but our patient had no such history and there are some other cases reported without any history of allergy too. $^{3}$ Exact etiology of the condition remains unclear as remained in our case. ${ }^{4,5}$

The initial symptoms, physical examination, and urinalysis may suggest a diagnosis of ureteric / renal calculus or urinary tract infection (UTI). Ultrasound and CT scan findings point towards the possibility of potential urothelial neoplasia. ${ }^{5,7}$ In our case, initially the provisional diagnosis was going in favour of right ureteric calculus or UTI. As recommended in the literature, this necessitates surgical urological intervention. Excision of the narrow segment is both diagnostic as well as therapeutic in this case. An open surgical excision or laparoscopic approach can be used to excise the narrow portion with end-to-end anastomosis of the remaining ureter. $^{3}$ It is curative in most cases 
although treatment with corticosteroids and antihistamines has been used in some cases. In case of any known triggering factor, eliminating the source may help. The management algorithm of our patient was according to the recent recommendations and literature available.

This patient had a normal eosinophil level in peripheral blood. The limitation we encountered during the management was the assessment of serum complements and immunoglobulin ( $\mathrm{IgG}$, $\operatorname{IgE})$ levels. Literature suggests that elevation of eosinophil and decrease in level of complements and immunoglobulin (IgG, IgE) may be related to allergy and autoimmune conditions.

The diagnostic confirmation is mainly based on the histopathological examination of the excised specimen. ${ }^{4}$ We also concluded the diagnosis postoperatively based on the finding suggested by histopathology report. Although the majority of luminal obliterated segmental lesions of the ureter are neoplastic and non-neoplastic inflammatory processes as seen in this case may occur in the ureter, causing diagnostic confusion with true neoplasms. ${ }^{5}$ As reviewed by Spark et al, two nonneoplastic entities of the ureter, eosinophilic and idiopathic segmental ureteritis causes ureteral obstruction due to mural involvement. However, the eosinophilic ureteritis never forms an intraluminal mass and is not associated with mucosal ulceration. ${ }^{3}$ Marked eosinophilic infiltration of the sub mucosa is the typical microscopic finding in eosinophilic ureteritis and this universal finding was consistent with that of our patient. ${ }^{4}$

\section{CONCLUSIONS}

Eosinophilic ureteritis is a rare non-neoplastic inflammatory process of the ureter that can mimic a renal / ureteric calculus or UTI. Malignancy must be ruled out and excision of the narrow segment with avoidance of the triggering factor is curative.

To cite this article: Bajracharya UB, Sah AK, Baral H, Sigdel G, Shrestha GK, Adhikari R. Eosinophilic Ureteritis Causing Ureterohydronephrosis: A Case Report. MJSBH. 2021;20(2):170-2.

Conflict of Interest: None declared

\section{REFERENCES}

1. Smith RC, Levine J, Dalrymple NC, Barish M, Rosenfield AT. Acute flank pain: A modern approach to diagnosis and management. Semin Ultrasound CT MRI. 1999 Apr 1;20(2):108-35. DOI: 10.1016/s0887-2171(99)90042-2

2. Singh JC, Karnik S V, Gopalakrishnan G. Eosinophilic ureteritis presenting as bilateral upper ureteric strictures. Scand J Urol Nephrol. 2004;38(3):260-2. DOI: 10.1080/00365590410025523

3. Spark RP, Gleason DM, DeBenedetti CD, Gigax JH. Is eosinophilic ureteritis an entity? 2 case reports and review. J Urol. 1991 Jun 1;145(6):1256-60. DOI: 10.1016/s0022-5347(17)38594-4

4. Joo M, Chang SH, Kim H, Lee KC, Ro JY. Idiopathic segmental ureteritis, misdiagnosed as ureteral cancer preoperatively: A case report with literature review. Pathol Int. 2010 Dec;60(12):779-83. DOI: 10.1111/j. 1440-1827.2010.02598.x

5. Sergeant G, Slabbaert K, Werbrouck P. Recurrent flank pain caused by eosinophilic ureteritis mimicking urinary stone disease: A case report. Int Urol Nephrol. 2004 Mar 1;36(1):23-5. DOI: 10.1023/b:urol.0000032678.84766.70

6. Platt ML, Kiesling VJ, Vaccaro JA. Eosinophilic ureteritis associated with eosinophilic cholangitis: A case report. J Urol. 1990;144(1):127-9. DOI: 10.1016/s0022-5347(17)39389-8

7. Stormont G, Makari J, Bedrnicek JB, Berrondo C. Eosinophilic Ureteritis in a Child With Ureteropelvic Junction Obstruction and Vesicoureteral Reflux. Cureus. 2020 Jul 15;12(7). DOI:10.7759/cureus.9214 